\title{
Electrically-activated catheter using polypyrrole actuators: cycling effects
}

\author{
Tissaphern Mirfakhrai, Tina Shoa, and Niloofar Fekri, and John D. Madden \\ Department of Electrical and Computer Engineering and Advanced Materials and Process \\ Engineering Laboratory, University of British Columbia, \\ Vancouver BC, V6T 1Z4, Canada
}

\begin{abstract}
The effect of cycling on charge-storage, actuation and sensing behavior of a polypyrrole is studied, having its application for an electroactive catheter in mind. It is shown that the electrochemical capacitance of a polypyrrole film decreases by about $15 \%$ over the course of 100 cycles, while the per cycle rate of this decrease drops by $75 \%$ between the first and the last ten cycles, implying that a steady-state value may exist. The decrease in capacitance is shown to have a significant effect on actuation strain. In order to achieve a more constant capacitance and more robust actuation performance, it is proposed to pre-cycle the potential of the film to exhaust the effect of processes that contribute to the decrease in capacitance and allow it to reach a more constant value. The ability of a polypyrrole film to generate currents corresponding to applied external load during actuation is verified and the cycle life time of such a sensor is studied. It is shown that after an initial decrease, the sensor current reaches a steady-state value as well, and maintains that value at least over 5600 cycles.
\end{abstract}

\section{INTRODUCTION AND BACKGROUND}

Natural muscle incorporates all the elements of a feedback loop needed to accurately control position and force. All the components of the feedback loop are built using a homogenous network of large biomolecules such as actin-myosin filaments that generate force and displacement, and muscle spindle fibers that measure displacement and velocity. Conducting polymers comprise the only class of artificial materials from which all of the same feedback loop components can be manufactured. Conducting polymer actuators, force sensors, and transistors have all been built [1-6]. In fact, conducting polymers can perform many other functions that nature has evolved over billions of years. Conducting polymer capacitors can store energy, and conducting polymer light sensors or chemical sensors can act as artificial eyes and noses, respectively, to list just a few.

Conducting polymer actuators have demonstrated high stresses (up to tens of MPa) and reasonable strains (typically 1 to $6 \%$ and as much as 15 to $20 \%$ ) [7,8]. Using thin actuators, highly mobile ions, and high actuation potentials, strain rates above $10 \% / \mathrm{s}$ have been observed $[19,20]$. At the higher strain rates, a peak power to mass ratio of $150 \mathrm{~W} / \mathrm{kg}$ was observed, matching the power to mass ratio of mammalian muscle [9]. Meanwhile, mechanical force sensors made of conducting polymers have also been reported. These can be in the form of strain gages or charge-based sensors [10-12]. In a strain gage, the resistance changes as the gage is stretched. Thus, a current must be passing through the sensor to enable it to sense a force. The advantage of a charge-based sensor is that it can measure a change in mechanical force without needing to run an auxiliary current. This helps preserve energy. Another advantage of charge based sensing is that in this way a polypyrrole film can sense forces acting on it while actuating. This self-sensing ability is a great property that creates a further similarity with natural muscles, where sensors of force are built into muscle units themselves. The self-sensing property can have many applications, especially in biomimetic and biomedical applications.

Because of biocompatibility and ease of preparation of polypyrrole, it is often chosen for biological applications such as drug-delivery applications [16]. Studies on polypyrrole have shown no evidence of acute and subacute toxicity [13]. Also, studies investigating some of the basic biocompatibility issues

Electroactive Polymer Actuators and Devices (EAPAD) 2009, edited by Yoseph Bar-Cohen, Thomas Wallmersperger, Proc. of SPIE Vol. 7287, 72871I - (c) 2009 SPIE · CCC code: 0277-786X/09/\$18 · doi: 10.1117/12.816056 
involved in using PPy as a blood-contacting biomaterial shown that PPy-coated polyester fabrics do not cause hemolysis nor did they alter the blood coagulation properties, therefore highlighting the potential of PPy-coated fabrics in cardiovascular applications [14].

We are currently investigating an active catheter using polypyrrole actuators to maneuver inside arteries for intravascular medical procedures [17]. Figure 1 shows a schematic of the design. Steering such a catheter in arteries requires reliable creation of actuation strains of about $6 \%$ in polypyrrole. Actuation has not been reliably demonstrated when blood itself has been used as the electrolyte. Therefore, it has been suggested that an aqueous electrolyte is used rather than using the blood itself as the electrolyte. Considerations about the safety of the patient require that this electrolyte is encapsulated from the patient's blood. Even when encapsulated and sealed from the blood stream, certain requirements and limits apply for the strain and excitation potentials. At potential higher than $+0.8 \mathrm{~V}$ or lower than $-0.4 \mathrm{~V}$ versus $\mathrm{Ag} / \mathrm{AgCl}$ reference electrode, the polymer volumetric capacitance dramatically decreases within a few cycles [18]. This suggests that polypyrrole may become electrochemically unstable at those potentials. Besides, the active catheter must have an extended cycle life of minimum 50000 cycles. Therefore it is important to study the effect of active cycling on actuation and the capability of the actuator to contain electric charge, which is responsible for actuation.

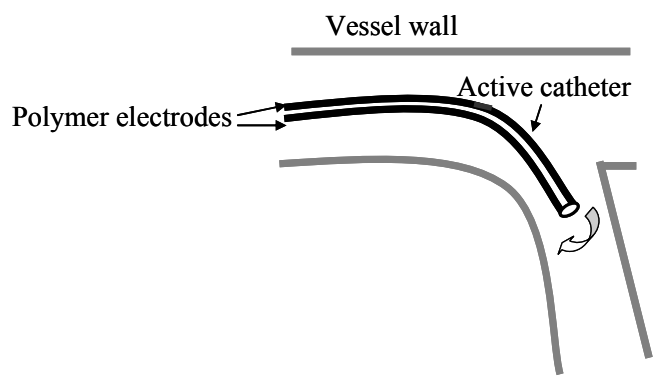

Figure 1. Schematic of the active catheter steered inside a blood vessel

In this paper, we study the effect of cycling on the charge-storage and actuation of a polypyrrole actuator by applying square waves and triangular waves (cyclic voltammograms) to it, with the ultimate application to an electroactive catheter in mind. The effect of cycling on charge storage capacitance and actuation strain is studied. The polypyrrole film is also shown to work as a mechanical force sensor during actuation. In order to study the life time of the film as a sensor, the effect of cycling the force acting on it for a few thousands of cycles is studied.

\section{METHODS}

Polypyrrole film was electrochemically deposited onto glassy carbon substrate. Polypyrrole deposition was done by polymerizing the pyrrole monomer through electrochemical oxidation using the method of Yamaura et al. [15]. The electrolytic solution is composed of $0.06 \mathrm{M}$ distilled pyrrole, $0.05 \mathrm{M}$ tetraethylammonium hexafluorophosphate and $1 \%$ of solution volume distilled water in propylene carbonate (from Sigma-Aldrich). The reaction occurs at a constant current of $0.125 \mathrm{~mA} / \mathrm{cm}^{2}$ and a temperature of $-33^{\circ} \mathrm{C}$ for 8 hours to obtain a thickness of approximately $10-12 \mu \mathrm{m}$.

A sample of polypyrrole film with a length of $6 \mathrm{~mm}$, width of $4 \mathrm{~mm}$, and thickness of about $12 \mu \mathrm{m}$ was placed as the WE in an electrochemical cell, mounted in an electromechanical test setup made by Aurora Scientific Inc. The film was held between a fixed Teflon clamp at the bottom and a moving clamp at the top (Figure 2). The moving clamp was used to vary the applied load on the polymer, or to measure the actuation strain while the load was kept constant. Electrical contact to the polymer was also made at the moving clamp, which was kept at all times above the electrolyte surface. The film was then submersed in an electrolyte comprising of a $1 \mathrm{M}$ solution of sodium hexafluorophosphate in deionized water. A piece of AvCarb T75 carbon fiber paper was used as the counter electrode (CE), with dimensions (and capacitance) much larger than that of the polypyrrole film. The reference electrode (RE) was $\mathrm{Ag} / \mathrm{AgCl}$ in $3 \mathrm{M} \mathrm{NaCl}$, as provided by bioanalytical.com. 


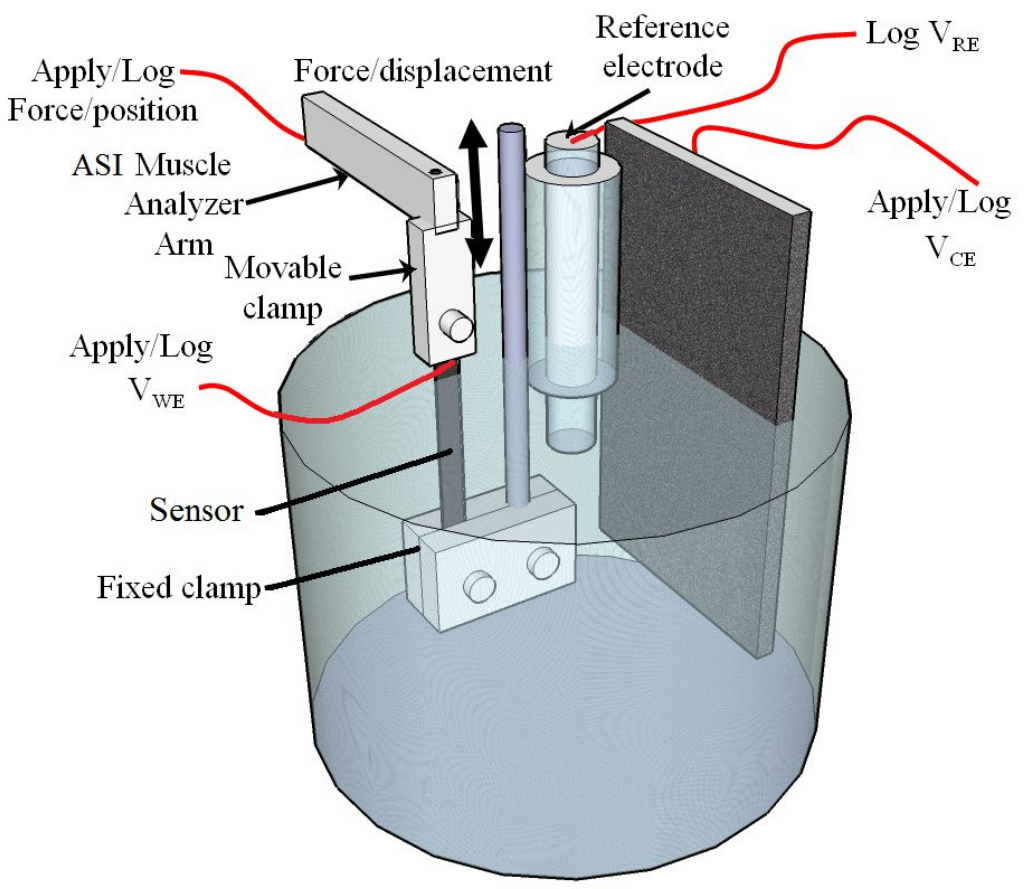

Figure 2: The electromechanical test apparatus and the electrochemical test cell

\section{EFFECT OF CYCLING ON CHARGE STORAGE AND CAPACITANCE}

The electrochemical capacitance of the film was estimated using cyclic voltammetry. Two sets of measurements were performed, one at scan rate of $8 \mathrm{mV} / \mathrm{s}$ and another at a scan rate of $0.8 \mathrm{mV} / \mathrm{s}$. In both cases the oxidation state of the polymer film was stabilized at around $0 \mathrm{~V}$ vs. RE for half an hour before the experiment was started. The resulting cyclic voltammograms are plotted in Figure 3a. The gravimetric capacitance is plotted as a function of cycle in Figure $3 \mathrm{~b}$.

As can be seen in Figure 3b, the capacitance at the higher scan rate is smaller. This is expected, because at higher scan rates the ions are given less time to penetrate into the polymer, and hence charge is effectively stored in a smaller volume of polymer. However, the relationship between capacitance and cycling seems to be the same at both scan rates. In both cases the capacitance initially decreases over about 15 cycles. After this initial phase, the capacitance seems to remain relatively constant between cycles 15 to 25 , before it starts to decline again. In the case of the $0.8 \mathrm{mV} / \mathrm{s}$ scan rate, the capacitance seems to have reached a steady-state value after about 70 cycles. At the faster scan rate, the capacitance changes from $268.9 \mathrm{~F} / \mathrm{g}$ to $244.4 \mathrm{~F} / \mathrm{g}$ over the course of 33 cycles. This corresponds to $9.1 \%$ change, or about $0.3 \%$ decrease per cycle on average. In the $0.8 \mathrm{mV} / \mathrm{s}$ experiment, the capacitance changes from 339.5 in the beginning to $288.1 \mathrm{~F} / \mathrm{g}$ after 100 cycles. This change corresponds to $15.1 \%$ decrease, or $0.151 \%$ decrease per cycle on average. While over the first 10 cycles the decrease rate is $0.43 \%$ per cycle, it drops to $0.12 \%$ over the course of the last 10 cycles. Similarly, in the $8 \mathrm{mV} / \mathrm{s}$ experiment, the rate of decrease in the capacitance goes from $0.49 \%$ per cycle in the first 10 cycles to $0.14 \%$ per cycle in the last 10 cycles.

Beside the number of cycles applied, other factors may contribute to the decrease in the capacitance. These include local changes in electronic and ionic conductivity due to gradual variations in oxidation state and chemical reactions with the electrolyte, which can exist during cycling, or even without cycling. 
It is possible that merely leaving the film in the NaPF6 electrolyte leads to slow degradation reactions that destroy the polymer, thus reducing its conjugation, conductivity and capacitance. In order to investigate this effect, an identical film from the same process was left in the cell for one hour in open circuit after its capacitance was measured using four $\mathrm{CV}$ cycles at $0.8 \mathrm{mV} / \mathrm{s}$. After one hour, $\mathrm{CV}$ cycles were repeated and the capacitance was measured again. The capacitance decreased by only $0.8 \%$, well within experimental error. To put this change into context, we should remember that one hour is the time needed to perform 24 $\mathrm{CV}$ cycles at $8 \mathrm{mV} / \mathrm{s}$ scan rate. Looking at Figure $3 \mathrm{~b}$, we can see that during the same time interval, the capacitance of film drops more than $8 \%$ when scanned at a rate of $8 \mathrm{mV} / \mathrm{s}$. It is therefore safe to conclude the cycling that changes the capacitance and not mere exposure to the electrolyte. Although exposure to water has been reported to decrease the polypyrrole conductivity and cause degradation [21,22], our results (not shown here) indicate that resting the polymer sample for half an hour after the capacitance has decreased during cycling experiments leads to more than $50 \%$ recovery of the lost capacitance. This implies that the change in capacitance is not only due to irreversible chemical degradation of the film.

Another part of this change in capacitance may be due to gradual change in the oxidation state of the polymer. The polypyrrole film is grown with a considerable number of $\mathrm{PF}_{6}^{-}$ions in it. These ions increase the conductivity of the film. At least in the initial CV cycles, it is possible that due to the very high concentration of ion in the polymer, the number of ions leaving during the reduction half-cycle is more than those entering during the oxidation half cycle. As a result, the ion content in the film, or perhaps parts of the film, will decrease, leading to a decrease in conductivity. The decrease in local conductivity means it takes more time to charge the film and so at a constant scan rate. This could be the reason for the decrease in capacitance.

It is also possible that the larger potential range used in the fast scan CVs can have an effect on the time it takes for the capacitance to reach the final stable value. Therefore, this effect must be carefully studied to find the optimal potential range resulting fastest stabilization of the capacitance. However, the CV plots in both cases show almost full charging of the film and no noticeable evidence of chemical reactions. Nevertheless, degradation processes may exist similar to those reported in polyaniline in aqueous solutions [23]. Lu et al. have studied the effect of cycling a PPy actuator on a helical wire backing [24]. It showed that the actuation strain drops by $\sim 60 \%$ when the polymer is actuated in propylene carbonate containing $0.25 \mathrm{M}$ Tetrabutylammonium hexafluorophosphate $\left(\mathrm{TBAPF}_{6}\right)$ for 2000 cycles. However the actuation strain in an ionic liquid (composed of 1-butyl-3-methyl imidazolium cation (BMIM+) and hexafluorophosphate anions $\left(\mathrm{PF}_{6^{-}}\right)$.) showed only $17 \%$ decrease during the course of 6000 cycles, and even that was recovered after leaving the polymer idle for one hour [24]. The mechanism of this reported recovery in strain can well be related to the recovery we have observed in the capacitance, despite the difference in the electrolytes.

Looking at the rates of capacitance change calculated above, it seems that the decrease rate after 33 cycles at $8 \mathrm{mV} / \mathrm{s}(0.14 \% /$ cycle $)$ is comparable with the rate of decrease after 100 cycles at $0.8 \mathrm{mV} / \mathrm{s}(0.12$ $\% /$ cycle). An experiment at the former scan rate takes substantially shorter time compared to an experiment at the latter rate. It is therefore advisable to cycle a film of polypyrrole at a relatively fast scan rate to exhaust the processes that decrease its capacitance. 


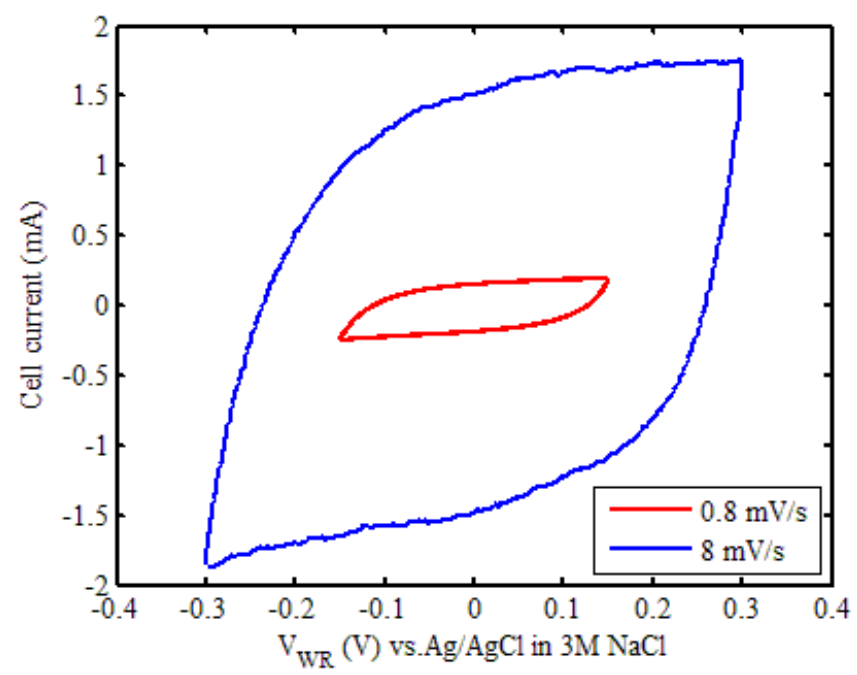

(a)

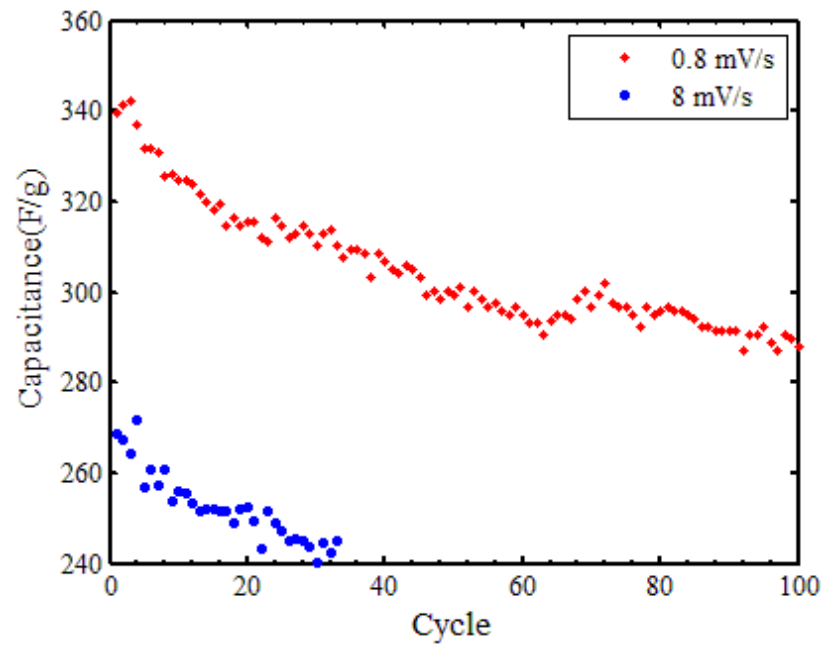

(b)

Figure 3: (a) cyclic voltammograms at $0.8 \mathrm{mV} / \mathrm{s}$ and $8 \mathrm{mV} / \mathrm{s}$ and (b) the measured capacitance at those two scan rate

\section{CYCLING EFFECTS IN ACTUATION}

The corresponding actuation strain during the cyclic voltammetry at $0.8 \mathrm{mV} / \mathrm{s}$ was measured. Figure $4 \mathrm{a}$ and $\mathrm{b}$ shows the peak-to-peak actuation strain during the first and the last four cycles, respectively. As can be seen in Figure 4a, there is a fast, clear trend in decrease in the peak-to-peak strain during the first four cycles. The mean actuation strain during the first four cycles is about $0.78 \%$, and the standard deviation from the mean is about $0.03 \%$. In comparison, the mean strain during the last four cycles is $0.54 \%$ with a standard deviation of only $0.003 \%$. Moreover, no clear increasing or decreasing trend can be seen in the peak-to-peak strain values and the strain seems to have stabilized. Comparing the values in Figure $4 \mathrm{a}$ and $\mathrm{b}$, it is found that the peak-to-peak strain changes from $0.83 \%$ during the first actuation cycle to about $0.54 \%$ during the last. This is a decrease of about $35 \%$, more than twice as large as the decrease in the capacitance. This implies that the strain-to-charge ratio of the polymer film is decreasing during cycling. Figure 5 shows the strain-to-charge behavior of the film during the first four cycles (gray) and the last four 
cycles (black). It can be seen that in addition to 15-20\% drop in transferred charge, which can be attributed to the drop in capacitance, the strain-to-charge ratio has also dropped. Taking the dimensions of the film into account, it can be estimated that the strain-to-charge ratio has dropped from an average of $4.6 \times 10^{-11} \frac{\mathrm{m}^{3}}{\mathrm{C}}$ during the first four cycles to $4.1 \times 10^{-11} \frac{\mathrm{m}^{3}}{\mathrm{C}}$ during the last. It is possible that this change in strain-to-charge ratio is because of structural changes in the film due to actuation. Before cycling, the film structure is more compact. The ions trying to leave or enter the film therefore need to push their way through narrow pores, resulting in significant interactions between ions and polymer chains, as well as interactions between adjacent ions themselves. These interactions create large forces that result in significant mechanical deformation in the film and corresponding strains. Gradually, pores open to an extent where interactions between adjacent ions decrease and ions can move in and out of the polymer structure with much less interaction with the pore walls, that have now been pushed further apart. This leads to smaller forces and therefore, less mechanical deformation and strain.

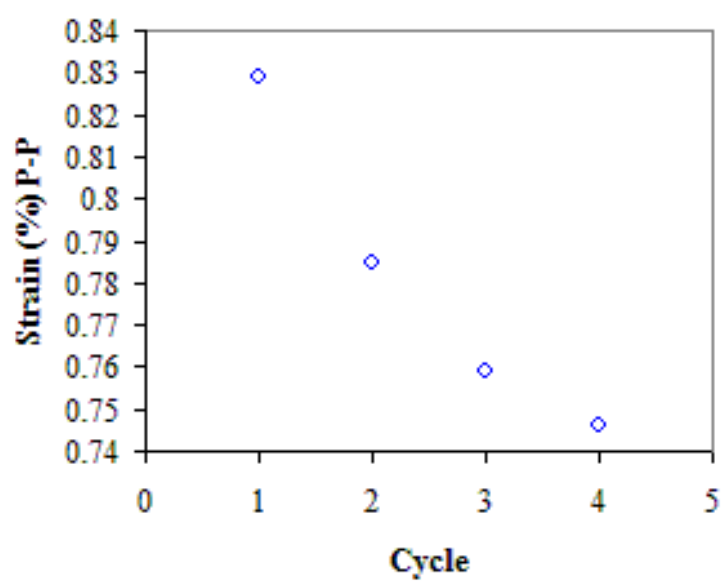

(a)

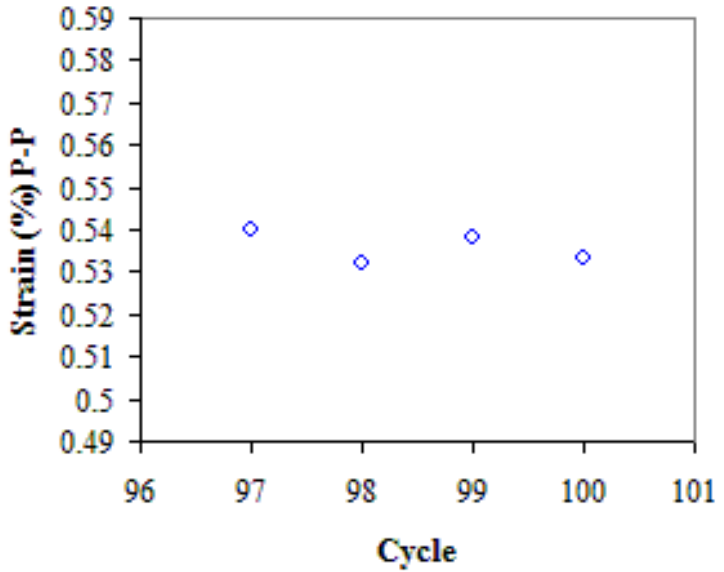

(b)

Figure 4: Actuation strain during (a) the first and (b) the last four cycles of the $\mathrm{CV}$ at $0.8 \mathrm{mV} / \mathrm{s}$.

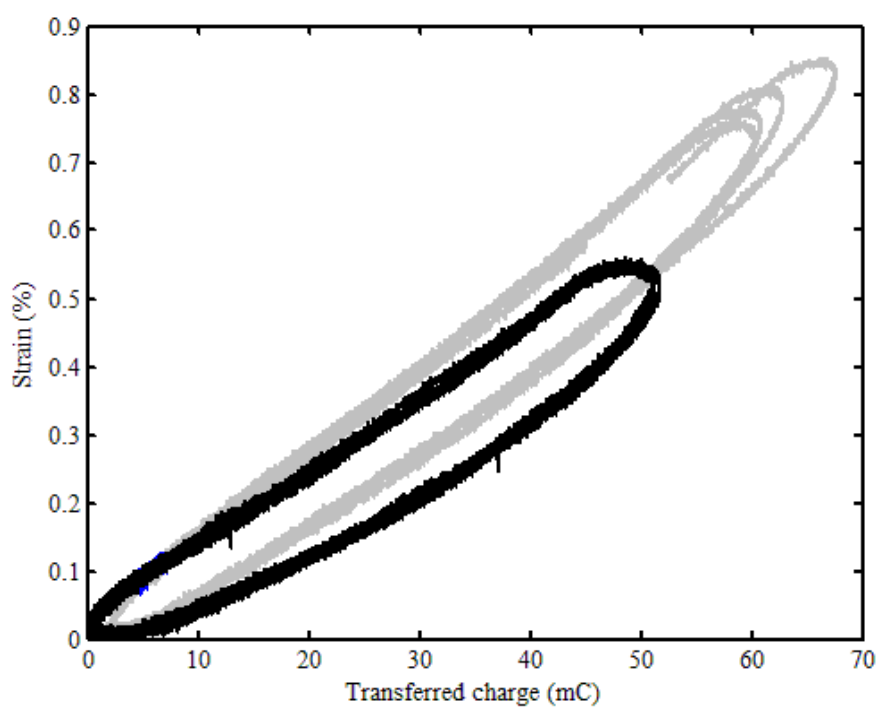

Figure 5: Strain as a function of the transferred charge during the first four cycles (gray) and the last four cycles (black) 


\section{MECHANICAL SELF-SENSING EFFECT}

One interesting property of polypyrrole actuators is that they can also be used to measure force $[11,12]$. Moreover, they can generate a response to the external force applied to them while the polymer film itself is actuating [5,6]. This effect can have broad applications and in particular for the catheter under investigation. The polypyrrole artificial muscles are used to navigate the catheter within the arteries and a sudden increase in the load applied to the polymer likely implies that the catheter has struck the arterial wall. Since any further pressure can lead to a puncture of arterial walls and internal bleeding, it will be of great assistance to the person operating the catheter to be provided with a feedback signal corresponding to the load applied on the artificial muscle.

It was previously shown that a film of polypyrrole can act as a force sensor $[11,12]$. To verify that it can also sense forces while actuating an experiment was conducted. A step in potential was applied to the film, while the external load on the polymer was cycled between 0 and $7 \mathrm{~g}$. The high-load half-cycle lasted 40 seconds, while the low-load half-cycle lasted 60 seconds. The resulting current from the combined effect of the potential and load change is plotted in Figure 6. The inset shows the variations in load. Using signal processing, it is possible to detect the rising and falling edges resulting from the changes in load. Such data can be used by the operator to detect interactions between the catheter and the arterial walls to avoid applying excessive pressure in the wrong direction. This can lead to safer operation.

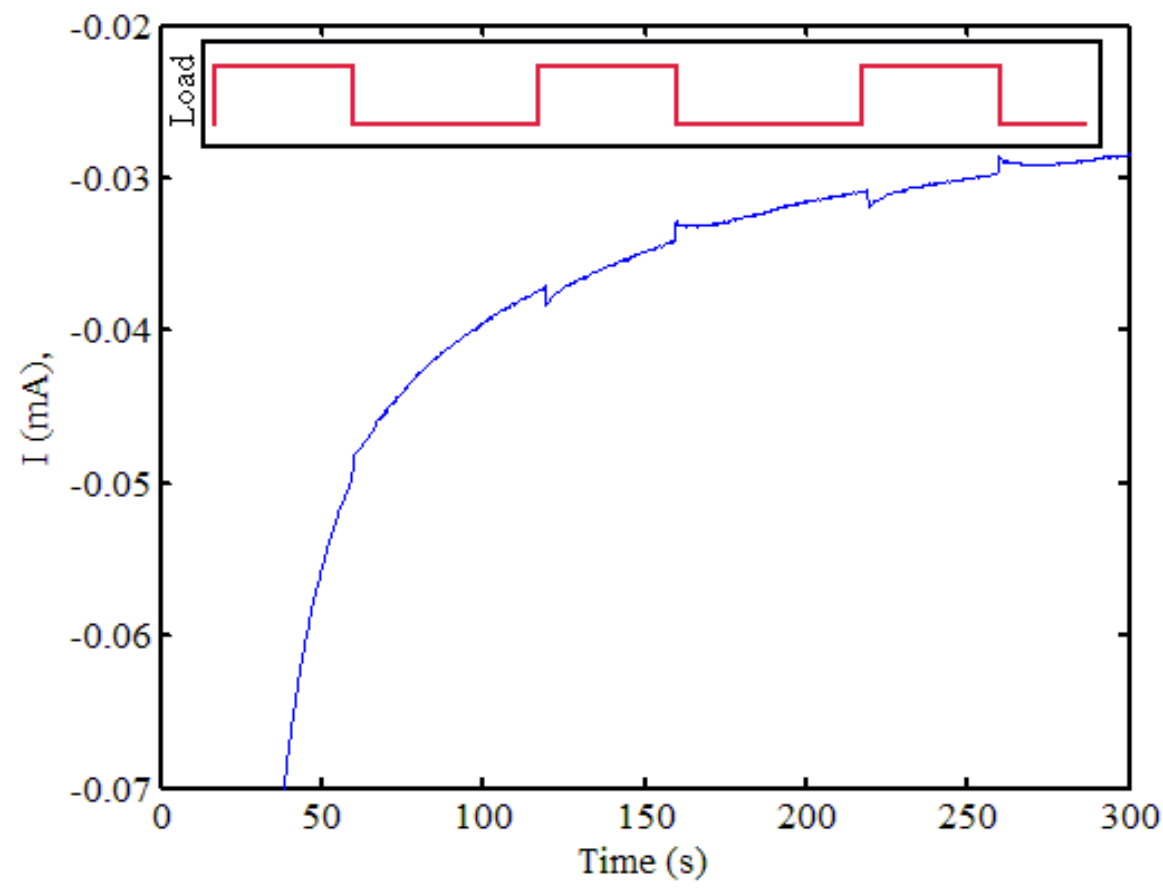

Figure 6: The combined effect of actuation and changing the external load on the current going through the cell.

\section{SENSOR LIFETIME STUDY}

If the self-sensing property of polypyrrole is to find application in the electroactive catheter, the cycle life of the polymer as a mechanical force sensor should match or exceed that of the actuator. To study the effect of cycling on the mechanical sensing properties, a test was conducted over the course of 15 hours. The applied external load on the polypyrrole sample was cycled in the form of a square wave similar to the plot in Figure 6 inset, but between $0.72 \mathrm{MPa}$ to $1.22 \mathrm{MPa}$ at a rate of 0.1 cycles per second. This amounts to about 5400 cycles. The polypyrrole sensor was biased in constant potential of $0 \mathrm{~V}$ versus a reference 
electrode of $\mathrm{Ag} / \mathrm{AgCl}$ in $3 \mathrm{M} \mathrm{NaCl}$. The amplitude of the sense current in response to this mechanical load input is plotted in Figure 7. It can be seen that the sense current amplitude starts at $0.5 \mu \mathrm{A}$. It then starts to drop as the load is cycled until after 5 hours it reaches a plateau of nearly $0.27 \mu \mathrm{A}$. Further cycling for 10 more hours seems to have little effect on the peak amplitude of the sense current and the performance of the sensor. The fact that the sensor response current reaches a steady-state value is reassuring. It means that the polypyrrole film can be used as a sensor for thousands of cycles.

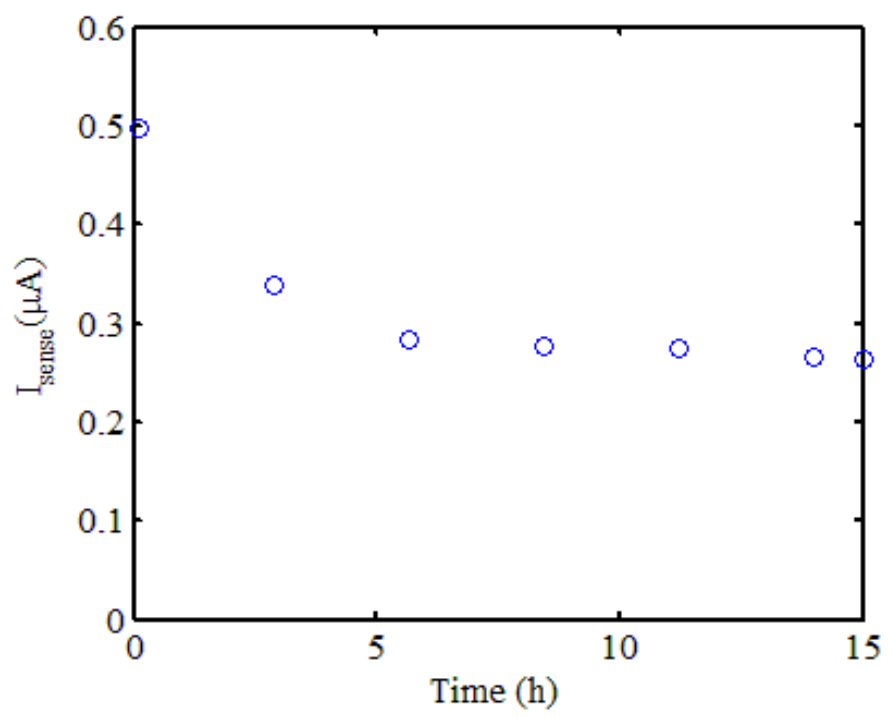

Figure 7: The amplitude of the sensing current of a polypyrrole film sensor at various times during a 15-hour lifetime study.

\section{CONCLUSIONS}

The effect of cycling on charge-storage, actuation and sensing behavior of polypyrrole was studied, having its application for an electroactive catheter in mind. It was shown that the electrochemical charge-storage capacitance of a polypyrrole film deceases by more than $15 \%$ over the course of 100 cycles, while the decrease rate per cycle drops by $75 \%$ between the first and the last ten cycles, implying that it may be reaching a steady-state value. It was proposed that pre-cycling can therefore exhaust this effect and lead to a more constant capacitance. Actuation performance during cycling was related to charge storage. Therefore, it was suggested that stabilizing the capacitance could improve actuation performance. Selfsensing ability of a polypyrrole film during actuation was verified and the cycle life time of such a sensor was studied. It was shown that the sensor current reaches a steady-state value as well, and maintains that value at least over 5600 cycles.

\section{REFERENCES}

[1] Madden, J. D., Madden, P. G., Hunter, I. W., "Polypyrrole actuators: Modelling and performance," Proc. SPIE - The International Society for Optical Engineering 4329, 2-83 (2001).

[2] Chiarelli, P., Della Santa, A., De Rossi, D., Mazzoldi, "Actuation properties of electrochemically driven polypyrrole free-standing films," Intelligent Material Systems and Structures 6, n 1, 32-37 (1995).

[3] Della Santa, A., De Rossi, D., Mazzoldi, "Performance and work capacity of a polypyrrole conducting polymer linear actuator," Synthetic Metals 90, n 2, 93-100, (1997).

[4] Okuzaki, H., Ishihara, M., Ashizawa, S., "Characteristics of conducting polymer transistors prepared by line patterning," Synthetic Metals 137, 947-948 (2003). 
[5] Otero, T. F., Cortes, M. T., “A sensing muscle,” Sensors and Actuators, B: Chemical 96, 152-156 (2003).

[6] Otero, T. F., Cortes, M. T., “Artificial muscles with tactile sensitivity,” Advanced Materials 15, 279282 (2003).

[7] Spinks, G. M., Wallace, G.G., Liu, L. and Zhou, D., "Conducting polymers electromechanical actuators and strain sensors," Macromolecular Symposia 192, 161-169 (2003).

[8] Anquetil, P. A., Yu, H.-H.; Madden, J. D.; Madden, Peter G.; Swager, T. M.; Hunter, I. W. "Thiophene-based conducting polymer molecular actuators," Proc. SPIE - The International Society for Optical Engineering 4695, 424-434 (2002).

[9] Madden, J.D.W., Vandesteeg, N. A., Anquetil, P. A.; Madden, P. G.A.; Takshi, A., Pytel, R. Z., Lafontaine, S. R., Wieringa, P. A.; Hunter, I. W. "Artificial muscle technology: Physical principles and naval prospects," IEEE Journal of Oceanic Engineering 29, n 3, Biology-Inspired Science and Technology for Autonomous Underwater Vehicles, p 706-728 (2004).

[10] Madden, J. D., "Conducting polymer actuators," $\mathrm{PhD}$ thesis Massachusetts Institute of Technology (2000).

[11] Wu, Y., Alici, G., Madden, J. D. W., Spinks, G. M., Wallace, G. G., "Soft mechanical sensors through reverse actuation in polypyrrole," Advanced functional materials 12, 3216 (2007).

[12] Takashima, W., Uesugi, T., Fukui, M., Kaneko M., Kaneto, K., "Mechanochemoelectrical effect of polyaniline film," Synthetic Metals 85, 1395 (1997).

[13] Wang, X. , Gu, X., Yuan, Ch., Chen, Sh., Zhang, P., Zhang, T., Yao, J., Chen, F., Chen, G., "Evaluation of biocompatibility of polypyrrole in vitro and in vivo," Journal of Biomedical Materials Research - Part A 68, n 3, 411-422 (2004).

[14] Zhang, Z., Roy, R., Dugre, F. J., Tessier, D., Dao, L. H., “In vitro $\quad$ biocompatibility study of electrically conductive polypyrrole-coated polyester fabrics," Journal of Biomedical Materials Research 57, n 1, 63-71 (2001).

[15] Yamaura, M., Hagiwara, T., Iwata, K., "Enhancement of electrical conductivity of polypyrrole film by stretching: counter ion effect," Synthetic Metals 26, 209-224 (1988).

[16] George, P.M., Lavan, D. A., Burdick, J. A., Chen, Ching Y., Liang, E., Langer, R., "Electrically controlled drug delivery from biotin-doped conductive polypyrrole," Advanced Materials 18, n 5, 577581 (2006).

[17] Shoa, T., Madden J. D., Fekri, N., Munce, N.R., Yang, V.X.D., “Conducting Polymer Based Active Catheter for Minimally Invasive Interventions inside Arteries," Proc. IEEE Engineering in Medicine and Biology Society proceedings, 2063 - 2066 (2008).

[18] Shoa, T., Cole, M., Yang V.X.D., Madden J.D., " Polypyrrole actuator operating voltage limits in aqueous NaPF6," Proc. SPIE Smart Structures \& Materials/NDE, Electroactive Polymer Actuators and Devices (EAPAD) 652, 652421 (2007).

[19] Hara, S., Zama, T., Takashima, W., Kaneto, K., "Free-standing polypyrrole actuators with response rate of 10.8\% s -1," Synthetic Metals 149, n 2-3, 199-20 (2005).

[20] Ding, J., Liu, L., Spinks, G. M., Zhou, D., Wallace, G. G., Gillespie, J., "High performance conducting polymer actuators utilising a tubular geometry and helical wire interconnects," Synthetic Metals 138, n 3, 391-398 (2003).

[21] Liu, Y.C., Hwang, B.J., "Mechanism of conductivity decay of polypyrrole exposed to water and enhancement of conductivity stability of copper(I)-modified polypyrrole," Journal of Electroanalytical Chemistry 501, n 1-2, 100-106 (2001).

[22] Neoh, K.G., Young, T.T., Kang, E.T., Tan, K.L., "Structural and mechanical degradation of polypyrrole films due to aqueous media and heat treatment and the subsequent redoping characteristics," Journal of Applied Polymer Science 64, n 3, 519-526 (1997).

[23] Genies, E. M., Tsintavis C., "Electrochemical behaviour, chronocoulometric and kinetic study of the redox mechanism of polyaniline deposits," Journal of Electroanalytical Chemistry 200, 127-145, (1986).

[24] Lu, W., Fadeev, A.G., Qi, B., Smela, E., Mattes, B.R., Ding, J., Spinks, G.M., Mazurkiewicz, J., Zhou, D., Wallace, G.G., MacFarlane, D.R., Forsyth, S.A., Forsyth, M., "Use of ionic liquids for $\pi$ conjugated polymer electrochemical devices," Science 297, n 5583, 983-987 (2002) 